\title{
Thirty years of physical activity in oncology in Germany- from the birth of the first rehabilitative cancer sports group until today
}

\author{
Klaus Schüle \\ Received: 14 December 2012 / Accepted: 23 December 2012 /Published online: 12 January 2013 \\ (C) European Group for Research into Elderly and Physical Activity (EGREPA) 2013
}

\begin{abstract}
Since 1978, exercise and sport therapy for oncological patients is a research and education focus at the German Sport University of Cologne. Back then, the top priority for almost all oncological patients was to "rest". Therefore, uncharted territory was entered. Aside from acute care hospitals, rehabilitation hospitals especially for oncological patients exist in Germany. In terms of the rehabilitation chain, which consists of acute care hospital, rehabilitation center, and home-based training, and the aspect of exercise therapy for breast cancer patients, the following progress could be observed in the last 30 years: In the 1980 s, less than $50 \%$ of the breast cancer patients received supervised exercise therapy during their 2- to 3-week hospital stay. Today, the length of hospital stay was reduced to 4 to 6 days and some hospitals have gym equipment to increase strength and endurance. In the beginning of the $1980 \mathrm{~s}$, passive applications (physiotherapy, massages, bubble baths, short hikes) were still dominant. While the rehabilitation stay took 6 weeks back then, it was reduced to 3 weeks today. Further, mainly groupbased and active measures are applied today. The aim is to encourage and motivate patients to long-term physical exercise. In the meantime, mainly exercise and sports therapists are employed, in order to meet the holistic bio-psychosocial requirements. The first cancer sports groups for breast cancer patients in the aftercare were founded in 1981 in Germany. Today, nearly 1,000 groups exist. Breast cancer and 15 other entities are represented, however mainly women take part. In the early days, research focused on the influence of physical
\end{abstract}

\section{K. Schüle $(\bowtie)$}

Institute for Exercising Therapy, Prevention and Rehabilitation,

Am Sportpark Müngersdorf 6,

50933 Cologne, Germany

e-mail: schuele@dshs-koeln.de activities on psychosocial aspects. Lately the effects on molecular, biological, and immunological parameters are also examined, in order to explore canceroprotective mechanisms.

Keywords Cancer · Germany · Physical activity · Exercise · Rehabilitation

\section{Background}

“Are you sure that sport doesn't induce the development of metastasis?"

(Question of an oncologist in 1981 shortly before founding our first rehabilitative cancer sports group)

Rehabilitative sport groups for patients with heart attacks, artificial hip joints (TEP), or a number of other chronic diseases, were set up very early at the German Sport University Cologne. In 1980, also oncological patients were supposed to be included in our programs. First of all, breast cancer patients with a restricted arm/ shoulder mobility resulting from surgery were involved in the program. However, for most physicians, it was unthinkable to expose their patients to an extra physical activity program. "Rest" was still regarded as the supreme recommendation during and after oncological treatment.

\section{General conditions}

For a better understanding of the past 30 years, the general conditions of the rehabilitation process (acute hospital-rehabilitation center-residence) within the past three decades will be demonstrated and compared (Fig. 1). 


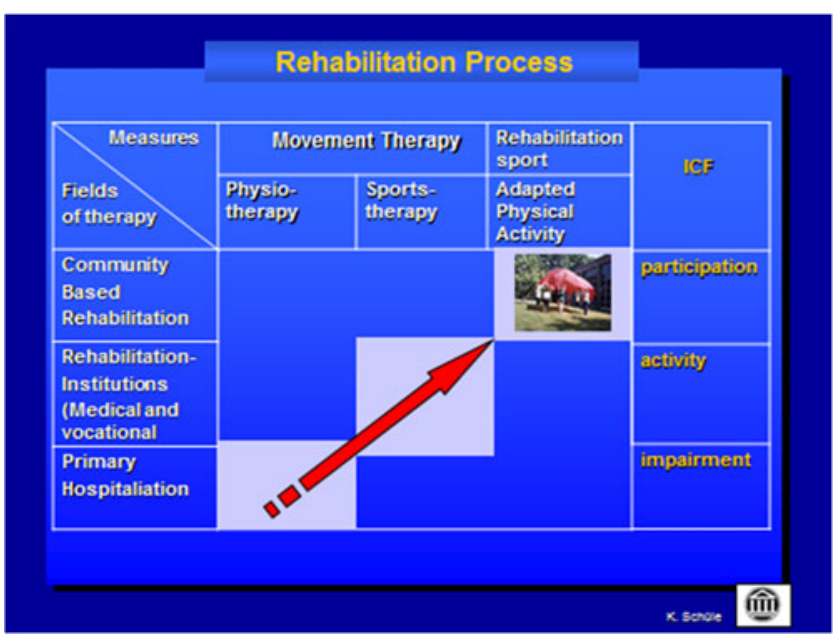

Fig. 1 The rehabilitation process

\section{Acute hospital}

In the initial phase (1980-1989), we mainly concentrated on breast cancer patients. Following surgery, which often involved total amputation and resection of $\mathrm{mm}$. pectoralis major and minor, a hospital stay of 2-3 weeks was common. Of course, back then, surgery-chemotherapy-radiotherapy were already part of the standard medical treatment options. Hormone therapy was still in its infancy.

Psycho-oncological care was primarily taken over by nurses or in some cases by physiotherapists. Psychooncology fought for recognition, since many physicians believed every dialog in principle was a small psychotherapeutic session.

What has changed in the past two decades?

In the last 20 years, medical diagnostics have improved; tumors are detected earlier. Surgical techniques are less radical, so that $60-70 \%$ of all surgeries today are breastsparing and most patients are able to leave the hospital within 1 week after surgery. Furthermore, hormone therapy has become a standard treatment. Regarding psychooncology, it seems to have established in the field of oncology.

Initially, not even half of all breast cancer patients received physical therapy despite the serious movement restrictions [3]. This was the reason for us to initiate an active rehabilitation that continued at the place of residence! In Cologne, patients received a regular and immediate physiotherapeutic care, which continued a few days later within a group setting in a gym. Also, patients in the aftercare were able to join the groups. As a result of this, even though movement impairments due to the dissection of axillary lymph nodes or even sentinel node dissection have decreased, still approx. $70 \%$ of the treated women were affected. Unfortunately, still not all women receive the necessary physical therapy within the first post-operative days! Finally, the personnel situation also changed in the last 20 years. Gradually, other movement therapists, namely sports therapists, are complementing the physiotherapists.

\section{Rehabilitation}

In the startup phase, the rehabilitative after care was still approved generously. Breast cancer patients were able to attend a 4-6-week "stabilization treatment" at a health resort yearly within the first 3 years after surgery. In 1981, for the first time, a rehabilitation procedure for cancer patients was performed (rehabilitation following in-patient treatment existed nationwide since 1977). Possibly, Germany is the leading nation when it comes to its rehabilitation system with its specialized clinics. However, several "health laws" emerged in order to save costs and therefore the rehabilitation period was shortened to 3 weeks without conducting any previous evaluations! Today, a second or even third stay at a rehabilitation center is difficult to enforce.

Conditions in the rehabilitation centers were very heterogeneous. There were some rehab centers with excellent fitness rooms, swimming pools, and small sport halls [6]. Other clinics had a scenic location however floors had to serve as gym rooms! The staff situation was similar. Back then, $60 \%$ of the therapeutic exercise offers where conducted by massage therapists or balneotherapists. Further $18 \%$ were undertaken by physiotherapists, $14 \%$ by gym teachers, and finally $2-3 \%$ by physical education teachers, mostly educated solely for school! [5].

Fortunately, the situation has changed in the following years. Today you can find swimming pools, appropriate sport halls and of course also specialized staff in the above mentioned clinics. In 1978, the first students majored in "rehabilitation and disabled sports" at the DSHS in Cologne. Their main application areas are in the out-patient and inpatient rehabilitation!

\section{Measures}

Physical exercise offers have changed in almost all institutions: moving away from passive fango applications and thermal baths, towards active and holistic exercise programs. Unfortunately longer hikes are only rarely possible due to higher staff costs. Overall, the purely functional training of earlier times is replaced by the holistic biopsycho-social movement therapy, which is specified in so called evidence-based treatment modules. These are the "guidelines" of the German statutory pension insurance scheme (DRV-Bund), the main payers of medical rehabilitation. Thus, more than $60 \%$ of the current rehabilitation offers consist of therapeutic exercise interventions! 


\section{Place of residence}

After three of our sport students successfully completed their programs at the rehabilitation centers in 1980, some of the participating patients returned to Cologne and joined the ambulatory sport group at the DSHS. Based on the snowball principle, more and more breast cancer patients from Cologne joined the group, including patients from local support groups.

The further development is well-known. Together with the State Sport Association of North Rhine-Westphalia, a nationwide network was established and our activities were extended to other states. Every other year, all state sport associations and disabled sport associations are invited to a national meeting to discuss latest results. Our activities have been supported by the EU within the campaign "Europe Against Cancer".

In the second phase (1990-1999), the number of groups was increasing. The 500th group was set up in 1999 in Barby (Saxony-Anhalt, new eastern state of Germany). By 2011, approx. 900 groups existed in Germany (Fig. 2).

I was proud to build up the "Cologne Consensus" in 1991. We brought together all relevant organizations and professional groups, including sport associations, doctors, physical education teachers, the German Cancer Aid and the German Cancer Society etc., to develop a national education curriculum for trainers of the cancer sports groups. From then on, the qualification has been uniform nationwide [4].

\section{Cancer entities}

At first, we had some model sports groups with very different cancer entities in North Rhine-Westphalia. We established, for example, a very successful mixed group, consisting of laryngeal cancer patients and breast cancer patients, both survivors and co-survivors. Groups for colorectal cancer patients were not successful. Overall, patients with approx. 20 different cancer entities participated in these groups. However, over $90 \%$ were breast cancer patients. A

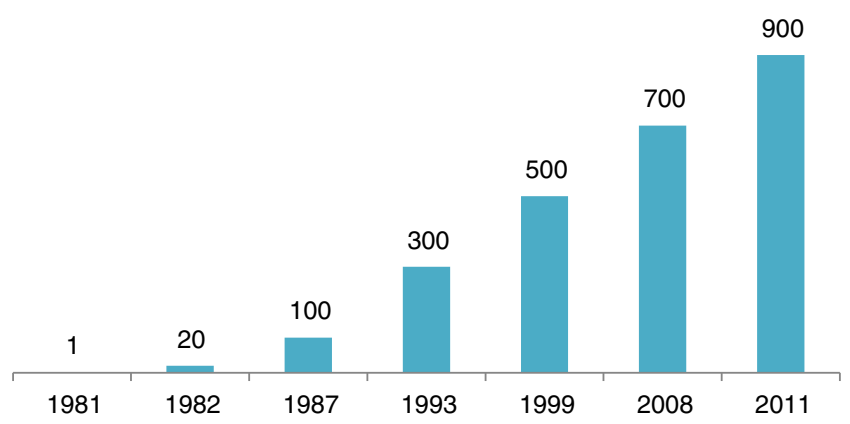

Fig. 2 Development of rehabilitative cancer sport groups in Germany (1981-2011) shift was promoted in the last 10 years, when Freerk Baumann implemented a prostate cancer project with the Cancer Society of North Rhine-Westphalia, the State Sport Association of North Rhine-Westphalia and the German Sport University Cologne. By 2012, over 40 rehabilitative sport groups particularly for prostate cancer patients were established in North Rhine-Westphalia.

\section{Research}

Our first investigations in rehab centers aimed at analyzing the current situation of breast cancer patients after surgery, with regard to their physical, psychological, and social wellbeing, i.e., with regard to their health-related quality of life.

First studies examining the effects of moderate endurance training on immunological parameters were conducted by Christiane Peters and Helmut Lötzerich from the German Sport University Cologne, together with Prof. Gerhard Uhlenbruck from the Immunological Institute of the University Hospital Cologne [2].

The assessment methods were not quite comparable to our present methods. Our questionnaires were self-made. In order to measure the range of motion in the shoulder joint, we used large goniometers that were specially produced by our precision mechanics. Load cells to measure muscle strength already existed, as did a bicycle ergometry in our Cardiovascular Institute. Unfortunately, these endurance performance tests had to be interrupted in many cases because - as the sport physician Richard Rost once put it — "the participants' ratio of size to weight was adverse!"

From the scientific point of view, we were not satisfied with the results yet, however a number of important practical experiences could be gained. Whenever, a woman revealed an impaired range of motion in the shoulder area of the operated side, for example, one could easily predict

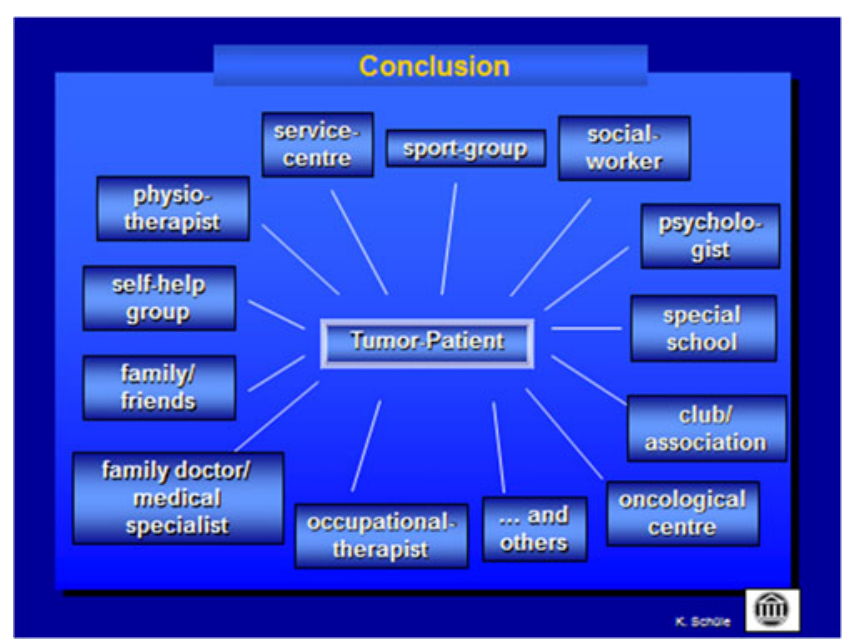

Fig. 3 Different institutions and disciplines have to work together 
that her quality of life was poorer than in someone who did not experience the restriction. When applying the very well-evaluated EORTC questionnaire on health-related quality of life today, the conclusion will be very similar. In other words, improved assessment methods do not necessarily result in more meaningful or applicationoriented results.

\section{Conclusion}

Today, assessment instruments are much better standardized and well evaluated, which now enables a valid comparison of similar issues and research.

The beginning of this third phase (2000-2010) was characterized by a spirit of optimism. Physicians were starting to get interested in our work and our bio-psycho-social approach with its "soft" results. We increasingly included cellular and molecular aspects. Changes in tumor markers, NK cells, T-cell subsets, etc., were meant to provide "hard" data.

A further exciting step was to work together with Freerk Baumann, my diploma and postgraduate student, who examined the effects of physical exercise in BMT patients [1]. Similar observations were reported by Fernando Dimeo a decade earlier in Freiburg and Berlin.

We must not forget some smaller studies investigating therapeutic exercise interventions in children during their rehabilitation in Bad Oexen or in palliative patients accommodated at the Mildred Scheel House in Cologne.

\section{Questions for the next 30 years}

Thirty years is still a short period of time to find definite answers regarding the importance and verification of physical activity and movement therapy in oncology. Thus, in my opinion, the following three questions represent the greatest challenge for future exercise experts:

1. Can physical activity prevent or minimize the occurrence of cancer? Does physical exercise act cancer protective in terms of prevention?

2. Can physical activity directly influence the tumor process, and therefore repress cancer (regression) or extend survival?

3. To what extent can physical activity influence (healthrelated) quality of life in cancer patients?

Some investigations have already addressed some of these issues. However, the results are not sufficient and therefore evidence-based and reliable statements cannot yet be provided. In order to succeed, it is necessary that the different disciplines and institutions work together (Fig. 3).

Conflict of interest The author has no conflict of interest to declare.

\section{References}

1. Baumann FT, Kraut L, Schüle K, Bloch W, Fauser AA (2010) A controlled randomized study examining the effects of exercise therapy on patients undergoing haematopoietic stem cell transplantation. Bone marrow transplant 45(2):355-362

2. Peters C, Lötzerich H, Hoff G, Niemeier B, Schüle K, Uhlenbruck G (1994) Influence of endurance training on the natural cytotoxicity in cancer patients. Int J Sports Med 15:359

3. Schüle K (1983) Zum Stellenwert von Sport- und Bewegungstherapie bei Patientinnen mit Brust- und Unterleibskrebs. Rehabilitation 22 (1):36-39

4. Schüle K (1999) Ausbildungsinhalte "Sport in der Krebsnachsorge" für Übungsleiter wurde bestätigt. Forum DKG 14 (1)

5. Schüle K, Deimel H (1984) Sport- und Bewegungstherapie - eine empirische Erhebung zum Sportlehrerbedarf. Öff Gesundh-Wesen 46(12):603-607

6. Schüle K, Hamacher F, Booz S (1983) Sport- und Bewegungstherapie im Rahmen einer onkologischen Nachsorgekur. Öff Gesundh-Wesen 45(4):191-196 